\title{
Structure and phase equilibria of the Widom-Rowlinson model
}

\author{
J.M. Brader ${ }^{1}$ and R.L.C. Vink ${ }^{2}$ \\ ${ }^{1}$ Fachbereich Physik, Universität Konstanz, D-78457 Konstanz, Germany \\ ${ }^{2}$ Institut für Theoretische Physik, Heinrich-Heine-Universität Düsseldorf, \\ Universitätsstraße 1, 40225 Düsseldorf, Germany
}

(Dated: November 9, 2018)

\begin{abstract}
The Widom-Rowlinson model plays an important role in the statistical mechanics of second order phase transitions and yet there currently exists no theoretical approach capable of accurately predicting both the microscopic structure and phase equilibria. We address this issue using computer simulation, density functional theory and integral equation theory. A detailed study of the pair correlation functions obtained from computer simulation motivates a closure of the Ornstein-Zernike equations which gives a good description of the pair structure and locates the critical point to an accuracy of $2 \%$.
\end{abstract}

PACS numbers: 61.20.Gy, 61.20.Ja, 64.60.Fr, 05.20.Jj

\section{INTRODUCTION}

The Widom-Rowlinson (WR) model [1, 2] is the simplest model which correctly captures the phenomenology of fluid-fluid demixing for systems interacting via short range forces and is therefore of fundamental importance in the theory of fluids. In particular, the model undergoes phase separation at sufficiently high density with a critical point which belongs to the Ising universality class [3]. The model can be regarded as a non-additive hard-sphere mixture in which like species do not interact but unlike species exhibit hard-sphere repulsion with a given collision diameter $\sigma$. Although there exist related WR-type models in which only the cross interaction is non-zero we will reserve the term WR model for that with a hard sphere interaction between unlike species.

Despite the simplicity of the interactions, an accurate theory of the bulk structure and thermodynamics of the WR model has proved elusive. The lowest order meanfield (MF) theory [1, 2] yields a crude description of the pair correlation functions and predicts a phase boundary between $A$ and $B$-rich phases for which the location of the critical point is in considerable error when compared to recent simulation estimates [4, [5, 6, 7, 8, 9]. However, to go beyond the lowest order theory appears to be a very demanding task. The first attempts at systematic improvement were made by incorporating information from higher order virial coefficients into the theory. In [10] virial, activity and cumulant expansions were considered. In 11] the virial series was studied for a WRtype model in which the $A B$ interaction is that of oriented hard cubes (chosen to facilitate the calculation of higher order virial coefficients). In neither case was reliable improvement obtained. Including additional virial coefficients was found to yield either results worse than the MF theory or no critical point, due to the appearance of multiple van der Waals loops in the free energy. The origin of these difficulties is the extremely erratic behaviour of the partial sums of the virial series. This issue was addressed in [12] in which the virial series of a WR type model with Gaussian $A B$ Mayer function was stud- ied. Even though the virial series for this model is much better behaved than for the WR case, Padé summation of terms up to 11th order in density was required to locate the critical point with reasonable accuracy. Convergence of the virial series in the neighbourhood of the critical point for the WR model appears to be much slower than for the Gaussian version. Although caution should be exercised when drawing conclusions regarding critical behaviour from low density expansions, it seems likely that the critical point of the WR model lies outside the radius of convergence of the virial series.

The correlation functions of the MF approximation in both the two-component and effective one-component versions of the model were studied in Ref. 13. The correlation functions were shown to display classical behaviour at the MF critical point and were proven to be exact in the limit of infinitely high dimensionality. A number of established integral equation theories have also been applied to the WR model [4] although none were able to account satisfactorily for the structure or phase boundary. Of the theories tested only the Percus-Yevick (PY) equation displayed a spinodal line, in all other cases (hypernetted chain, Martynov-Sarkisov, Rogers-Young and Ballone-Pastore-Galli-Gazzillo equations) the theory no longer converges in a region of the phase diagram, prior to divergence of the partial structure factors. Although $\mathrm{PY}$ is the best among the standard theories, it still gives a poor description of the structure and the critical points obtained from the spinodal and virial free energy are highly inconsistent. In order to address these difficulties Yethiraj and Stell (YS) developed an integral equation specifically for the WR model in which analytic expressions are derived for the direct correlation functions between like species $c_{A A}(r)$ and $c_{B B}(r)$ by resumming a class of diagrams which can be evaluated analytically [14, 15]. Unfortunately the YS equation strongly overestimates the structure and the pair correlation functions do not compare favourably with computer simulation.

In Ref. 16 a fundamental measures density functional was constructed for the WR model suitable for application to inhomogeneous situations. The theory predicts 
TABLE I: Properties of the statepoints at which pair correlation functions were obtained using computer simulation. Shown for each statepoint is the density $\rho_{A}$ of $A$ particles, the fugacity $z_{B}$ of $B$ particles, as well as the corresponding density $\rho_{B}$ of $B$ particles, the composition $x$, and the total density $\rho$.

\begin{tabular}{ccc|ccc}
\hline \hline statepoint & $\rho_{A}$ & $z_{B}$ & $\rho_{B}$ & $x$ & $\rho$ \\
\hline 1 & 0.1 & 0.8 & 0.5815 & 0.1467 & 0.6815 \\
2 & 0.3 & 0.8 & 0.3366 & 0.4713 & 0.6366 \\
3 & 0.4 & 0.8 & 0.2481 & 0.6172 & 0.6481 \\
4 & 0.5 & 0.8 & 0.1758 & 0.7399 & 0.6758 \\
5 & 0.6 & 0.8 & 0.1191 & 0.8344 & 0.7191 \\
6 & 0.7 & 0.8 & 0.0774 & 0.9004 & 0.7774 \\
\hline \hline
\end{tabular}

reasonable behaviour for the pair correlations and improves slightly on the predictions of the MF theory for the phase behaviour. However, the location of the critical point remains in error and thus does not permit investigation of the structure at statepoints in the vicinity of the simulation critical point. Despite all of these efforts it can be concluded that the overall level of accuracy of the existing theories remains unsatisfactory, given the fundamental nature of the model.

In this work we seek to develop a theory for the WR model which provides accurate predictions for the pair structure and which is able to locate the critical point to an acceptable level of accuracy. In order to base our approximations on firm foundations we will use simulation results for the pair structure to guide the construction of our theory. We focus in particular on the the total correlation function $h_{i j}(r)=g_{i j}(r)-1$, where $g_{i j}(r)$ are the radial distribution functions, the bridge function $b_{i j}(r)$ and the direct correlation function $c_{i j}(r)$. The paper is structured as follows: We begin by discussing the model in Section II In Section 【II, we present computer simulation results for $h_{i j}(r), c_{i j}(r)$ and $b_{i j}(r)$, focusing on high density statepoints off the critical line where the model is least well understood. These simulation results will act as motivation for our theoretical approaches in Section IV] Finally, we summarize our results and suggest possibilities for future work.

\section{THE WR MODEL}

The binary WR model is a symmetric mixture consisting of species $A$ and $B$. The interaction between likespecies is ideal, $\phi_{A A}(r)=\phi_{B B}(r)=0$, while unlikespecies interact via a hard-core potential of diameter $\sigma, \phi_{A B}(r)=\infty$ for $r<\sigma$, and zero otherwise. We henceforth take $\sigma$ as the unit of length. Above a certain critical density $\rho=\left(N_{A}+N_{B}\right) / V$, the WR model phase separates into two phases: one phase containing predominantly $A$ particles, and the other phase mostly $B$ particles. Here, $V$ is the volume, and $N_{A}\left(N_{B}\right)$ denotes the number of $A(B)$ particles in the system. Due to the symmetry of the model, the compositions

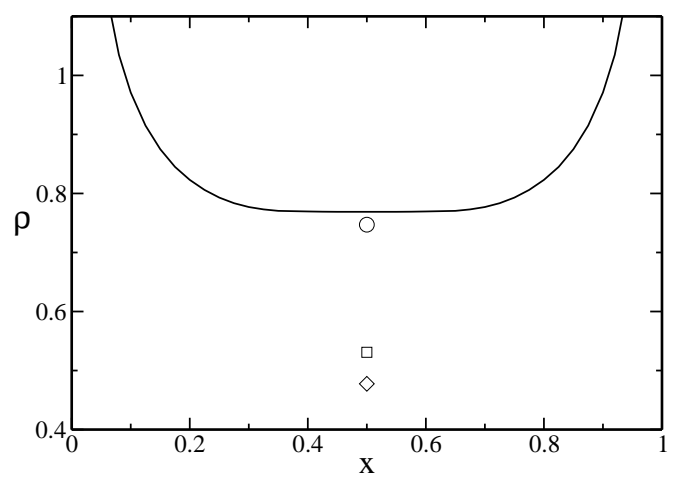

FIG. 1: The phase diagram of the WR model in $(x, \rho)$ representation. The line is the spinodal from the new integral equation closure, see Eq.(12), of this work. The circle marks the location of the critical point, as obtained in the simulations of Ref. 9. For comparison we also show the critical point predicted by the simple mean field theory of [1, 2] (diamond) and the density functional theory of Ref. 16 (square).

of the phases are given by $x$ and $1-x$, respectively, with $x=N_{A} /\left(N_{A}+N_{B}\right)$. At the critical point one has $x=1 / 2$. The phase diagram is thus conveniently represented in the $(x, \rho)$-plane, see Fig. 1, For densities $\rho>\rho_{\text {crit }}$, coexisting phases of composition $x$ and $1-x$ can be identified. The binodal, which is symmetric about the line $x=1 / 2$ in the $(x, \rho)$ representation, terminates at the critical point. In the mean field approximation the binodal exhibits a parabolic curvature around the critical point (recall the mean-field critical exponent of the order parameter $\beta=1 / 2$ ). The simplest mean-field estimate of the critical density is $\rho_{\text {crit }}=3 / 2 \pi=0.4775$ [1, 2], whereas the critical point of the PY spinodal lies at $\rho_{\text {crit }}=1.12$ [17, 18]. We emphasize that both of these approximations are mean field in character and exhibit classical critical exponents. In contrast, the best current simulation estimates for the critical density are $\rho_{\text {crit }}=0.7470(8)$ [7] and $\rho_{\text {crit }}=0.7486 \pm 0.0002[9]$, which, as was pointed out in the Introduction, is not accounted for by any theoretical approach.

A possible source for the discrepancy between simulation and theory is the fact that the WR model belongs to the universality class of the Ising model. For the Ising model, $\beta \approx 0.326$ [19], implying a flatter binodal. Computer simulations of the WR model indeed recover Ising critical behavior [ 5, 6, 7, 9]. However, in order to observe the pure Ising exponent $\beta$, the $(x, \rho)$ representation of the binodal is not the most convenient. For Ising systems, there is an additional singularity in the specific heat, governed by the critical exponent $\alpha \approx 0.109[19]$. In the $(x, \rho)$ representation, the curvature of the binodal is then described by the renormalized exponent $\beta^{\star}=\beta /(1-\alpha)$ [20] (in general, critical exponents become renormalized if the critical point is approached by varying a quantity 


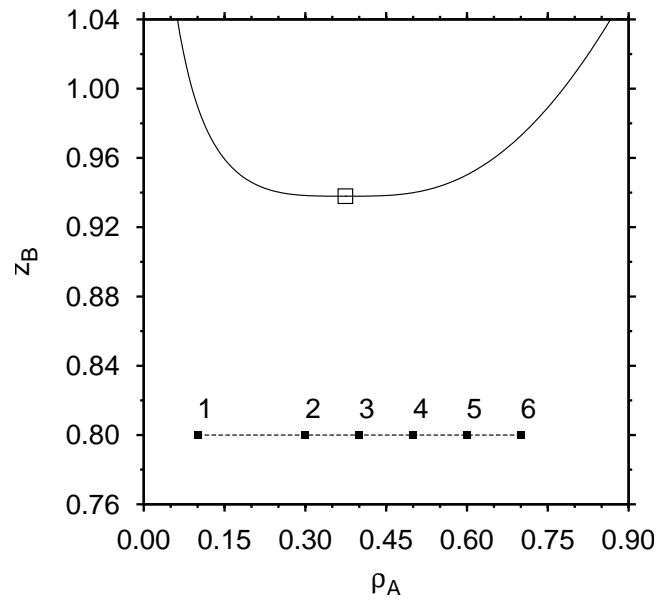

FIG. 2: Phase diagram of the WR model in $\left(\rho_{A}, z_{B}\right)$ representation. The solid curve shows the binodal; the open square marks the location of the critical point. The binodal was constructed using simulation data of Ref. 9 combined with finite size scaling, and so, on the scale of the above graph, accurately reflects the true thermodynamic limit form. The closed squares (labeled $1-6$ on the horizontal line in the one-phase region), mark the statepoints at which the simulations of this work were performed to obtain the pair correlation functions, see also Table 1

which is not a field variable). In order to observe the pure Ising exponent, the binodal should be represented in analogy to the (density,temperature) phase diagram of simple fluids. For the WR model, this would be a grandcanonical representation, where the density $\rho_{A}=N_{A} / V$ of $A$ species, and the fugacity $z_{B}$ of $B$ species, are the relevant variables [5, 9, 21] (the choice for $A$ or $B$ is of course arbitrary). Shown in Fig. 2 is the phase diagram in $\left(\rho_{A}, z_{B}\right)$ representation. The curvature of the binodal around the critical point, at $\rho_{A} \approx 0.3743$ and $z_{B} \approx 0.93791$, is now described by the pure Ising exponent $\beta[9]$. In contrast to the $(x, \rho)$ representation, the symmetry of the WR model is not obvious from the binodal of Fig. 2. The symmetry, of course, still exists. In the grand-canonical ensemble, it corresponds to the line of equal fugacities $z_{A}=z_{B}$. Note that for mean-field systems, the curvature of the binodal is not affected by the representation, since here $\alpha=0$.

\section{COMPUTER SIMULATIONS}

Fantoni et al. [8] have presented computer simulation results for $g_{i j}(r)$ and $c_{i j}(r)$ for several different values of $\rho$ along the symmetry line $x=1 / 2$. One of the most interesting conclusions arising from this work is that the Percus-Yevick condition, $c_{A B}(r)=0$ for all $r>1$, is satisfied to very high accuracy, even for statepoints approaching the critical point. However, it is not at all clear whether this property is also maintained off the symmetry line; the non-trivial cancellations which apparently occur in the diagrammatic expansion of $c_{i j}(r>1)$ on the symmetry line may no longer hold when $\rho_{A} \neq \rho_{B}$. Indeed, a theoretical approach which aims to describe phase separation must be able to accurately describe the change in pair structure as a function of $x$. As far as we are aware there exists no detailed study of the behaviour of the pair structure for $x \neq 1 / 2$.

\section{A. Simulation details}

Motivated by the above considerations, we have performed simulations for the off-symmetry statepoints given in Table [1. To simulate the off-symmetry statepoints $x \neq 1 / 2$, we use a quasi-grand canonical simulation ensemble, whereby the system volume $V$, the density of A particles $\rho_{A}$, and the fugacity $z_{B}$ of $B$ particles are fixed, while the number of $B$ particles fluctuates. The simulations are performed in cubic simulation boxes of edge $L=30$, using periodic boundary conditions in all $d=3$ directions. For the statepoints considered by us, this implies approximately 15,000 particles in each simulation box. To simulate efficiently, a cluster Monte Carlo move is used [22, 23]. We specialize to $z_{B}=0.8$, which is well below its critical value $z_{B, c r} \approx 0.93791$ [5, 9], and inside the one-phase region of the phase diagram, see Fig. 2. The density of the $A$ particles is then varied over the range $0.1-0.7$. For each statepoint, the average concentration $\rho_{B}$ of $B$ particles is measured, as well as the radial distribution functions $g_{i j}(r)$. The radial distribution functions are evaluated using a standard method [24], and averaged over approximately 5000 independent configurations. For each statepoint, this requires an investment of about $120 \mathrm{CPU}$ hours. A total of six distinct statepoints is considered. For each statepoint, the average concentrations $\rho_{A}$ and $\rho_{B}$, as well as the total concentration $\rho$, and the composition $x$, are listed in Table 【

\section{B. Analysis of simulation structure}

In Fig. 3 we show the total correlation functions $h_{i j}(r)$ for three of the considered statepoints. The correlations between like species are monotonic and exhibit no sign of any oscillatory packing behaviour. The increase of the $h_{i i}(r)$ as $r \rightarrow 0$ reflects the tendency of like species to overlap in order to maximize the free-volume and hence the entropy of the system. Equivalently, this clustering behaviour can be viewed as reflecting the attractive (many-body) depletion potential acting between spheres of species $A(B)$, induced by the sea of non-interacting spheres of species $B(A)$. For example, for small values of $x$ we expect $h_{A A}(r)=\exp \left[-\beta \phi_{d e p}(r)\right]-1$ and $c_{A A}(r)=\exp \left[-\beta \phi_{d e p}(r)\right]+\beta \phi_{d e p}(r)-1$, with depletion potential

$$
\beta \phi_{d e p}(r)=-\frac{4}{3} \pi \rho_{B}\left(1-\frac{3}{4} r+\frac{1}{16} r^{3}\right),
$$


for $r<2$, and zero otherwise. The cross correlation function $h_{A B}(r)$ is negative for all values of $r$ and indicates that in addition to the trivial hard core exclusion there is also an effective repulsion between species of opposite type. This is a consequence of the clustering of like particles and leads to the appealing picture of the bare hardsphere repulsion between species $A$ and $B$ being supplemented by a softly repulsive 'dressed' interaction describing particles shrouded by a cluster of like particles. Naturally, this effective interaction is of statistical origin and is therefore not to be taken too literally. The direct correlation functions are shown in Figs 4 and 5. To obtain the $c_{i j}(r)$ from our simulated $h_{i j}(r)$ we apply the method described in [8]. The Fourier transform $\tilde{h}_{i j}(k)$ yields $\tilde{c}_{i j}(k)$ via the Ornstein-Zernike relation. We then construct the difference $\tilde{\gamma}_{i j}(k)=\tilde{h}_{i j}(k)-\tilde{c}_{i j}(k)$ (the Fourier transform of a continuous function in real space) and transform back to get $\tilde{\gamma}_{i j}(k)$. We thus obtain $c_{i j}(r)$ from the difference $h_{i j}(r)-\gamma_{i j}(r)$. Both $c_{A A}(r)$ and $c_{B B}(r)$ display the same monotonic behaviour observed for the total correlation functions. The $c_{i i}(r)$ are shorter range functions than the corresponding $h_{i i}(r)$, as expected. In Figure 5 we show the cross correlation function $c_{A B}(r)$. The form of $c_{A B}(r)$ inside the core $(r<1)$ is quite different from the familiar case of an additive hard sphere system for which it is found that $c_{A B}^{h s}(0) \leq c_{A B}^{h s}\left(1^{-}\right)$at all densities. Outside the core, $r>1$, we find that the value of $c_{A B}(r)$ does not exceed $10^{-3}$ for any of the simulated statepoints. A quantity which is often of interest in liquid state integral equation theories are the bridge functions $b_{i j}(r)$. The bridge functions for a binary mixture interacting via pair potentials are defined by the relation

$$
h_{i j}(r)=e^{-\beta \phi_{i j}(r)+h_{i j}(r)-c_{i j}(r)+b_{i j}(r)}-1,
$$

where $\phi_{i j}(r)$ is the pair potential acting between species $i$ and $j$. In Fig. 6 we show the bridge functions obtained from our simulations. Note that data for $b_{i j}(r)$ for $r<1$ are not displayed as these are not required for calculations of the pair structure for systems with hard core interactions, see Eq.(2). While the function $b_{A B}(r)$ is of a rather simple form the functions $b_{i i}(r)$ take both positive and negative values.

We now consider the implications of the above for constructing approximate theories. The most important information to come from the simulations is that the PY approximation $c_{A B}(r>1)=0$ is satisfied to high accuracy at all of the considered statepoints. This fully supports and extends the findings of Fantoni et al. [8] and implies that the condition $c_{A B}(r>1)=0$ should be enforced in any approximate theory for this model. As the exact core condition $h_{A B}(r<1)=-1$ is also satisfied, this effectively reduces the problem to that of finding accurate approximations for $c_{A A}(r)$ and $c_{B B}(r)$. We note that our verification of the PY condition for the cross correlation functions invalidates the speculation in the discussion of Ref. 14. Here, it was suggested that a more accurate $c_{A B}(r>1)$ could be achieved by modeling the tail with a Yukawa function and adjust-

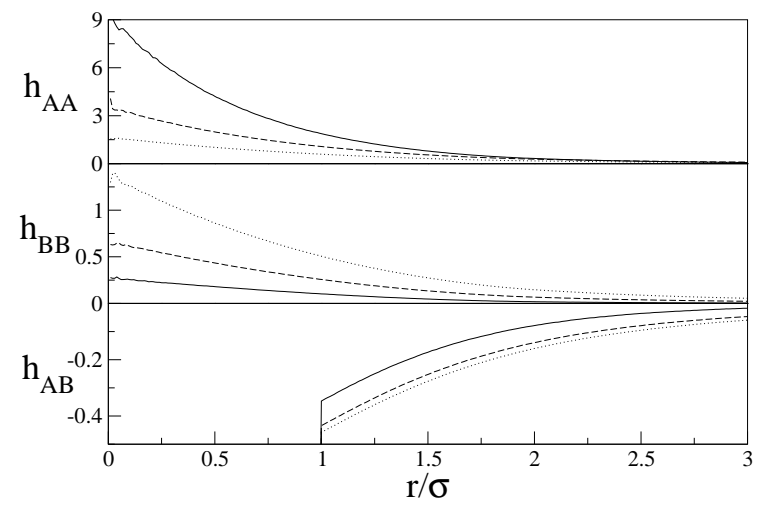

FIG. 3: The correlation functions $h_{i j}(r)$ from simulation for statepoints 6 (solid line), 4 (broken line) and 2 (dotted line) (see Table I). Note that $h_{A B}(r<1)=-1$.

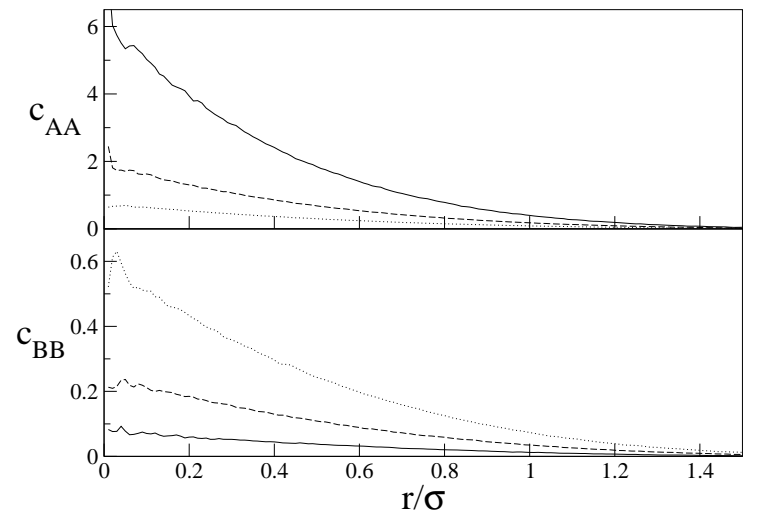

FIG. 4: The correlation functions $c_{A A}(r)$ and $c_{B B}(r)$ from simulation for statepoints 6 (solid line), 4 (broken line) and 2 (dotted line).

ing the free parameters to achieve thermodynamic self consistency. This program, while successful for the case of hard spheres, would apparently lead to no significant improvement over the simple PY approximation for the present model.

The direct correlation functions $c_{i i}(r)$ are of significantly shorter range than the $h_{i i}(r)$ and possess a relatively simple monotonic form. It may therefore be useful to model the $c_{i i}(r)$ by suitably chosen basis functions of finite range. By choosing finite range basis functions we naturally suppress the development of realistic critical exponents (the direct correlation functions are known to become long range at the critical point [25]). However, we do not necessarily restrict ourselves to mean field criticality in making this choice. The bridge functions between like species $b_{i i}(r)$ are very different from those of the hard 


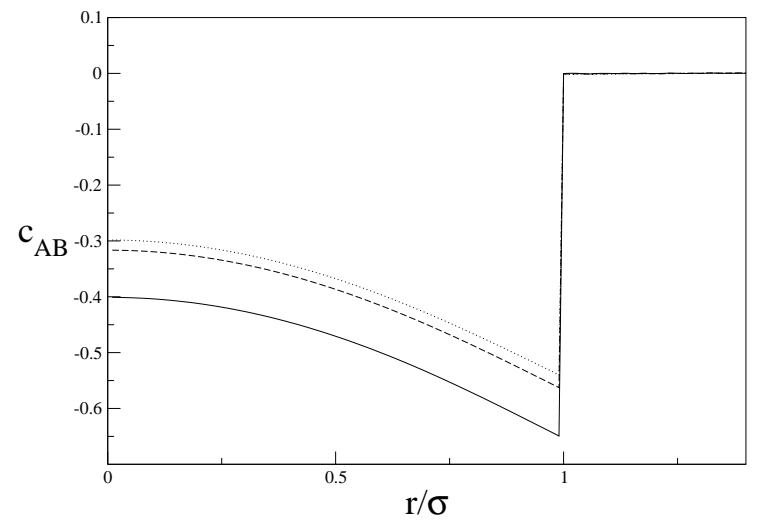

FIG. 5: The correlation function $c_{A B}(r)$ from simulation for statepoints 6 (solid line), 4 (broken line) and 2 (dotted line). $\left|c_{A B}(r>1)\right|<10^{-3}$ at all simulated statepoints.

sphere system [26] and exhibit regions of both positive and negative sign. The function $b_{A B}(r>1)$, although superficially similar to the hard sphere bridge function, is found to display a quite different functional form and cannot be reasonably fitted using the PY bridge function for hard spheres at any effective density. These findings suggest that modified-hyper-netted-chain (MHNC) type approximations [27], where universality of the hardsphere family of bridge functions is assumed, will not prove useful in this case. Indeed, the complex damped oscillatory form of the functions $b_{i i}(r)$ suggests that approaches aiming to directly approximate the bridge functions should be avoided.

\section{THEORETICAL APPROACHES}

The simulation results presented in the previous section indicate that a successful theory for the pair structure of the WR model may be constructed using the conditions $h_{A B}(r<1)=-1, c_{A B}(r>1)=0$ in combination with a suitable "ansatz" for the short range functions $c_{i i}(r)$. In this section we investigate this possibility. Our desire to identify suitable basis functions to describe $c_{i i}(r)$ leads us to consider a simple virial expansion based density functional approximation. This yields analytic results for the pair structure which, although only strictly valid at low density, actually give a reasonable account of the structure over the entire phase diagram. Modification of these results to incorporate the core condition leads to a new integral equation closure.

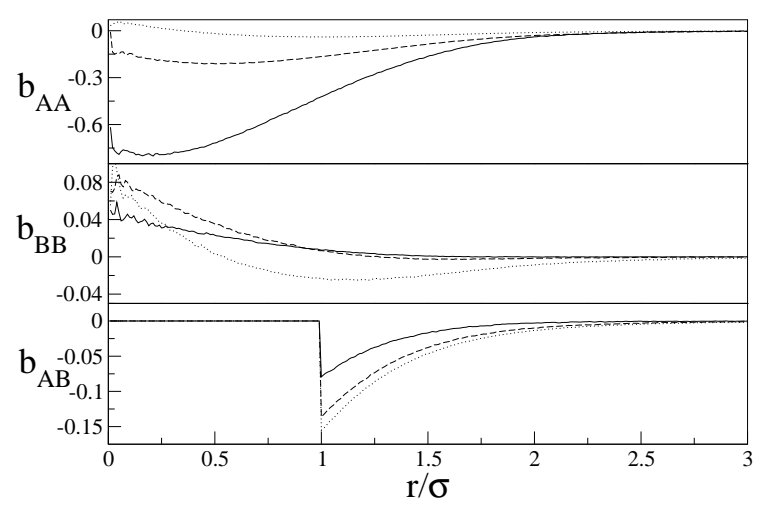

FIG. 6: The correlation functions $b_{i j}(r)$ from simulation for statepoints 6 (solid line), 4 (broken line) and 2 (dotted line).

\section{A. Density functional approach}

Density functional theory (DFT) is a formalism which enables the calculation of thermodynamic and structural properties of systems subject to spatial inhomogeneity 28]. A key result is the stationarity of the grand potential with respect to variations in the inhomogeneous density fields, $\delta \Omega / \delta \rho_{i}(\mathbf{r})=0$, where $i$ labels the species. Given an explicit functional, this condition yields a set of coupled equations for the $\rho_{i}(\mathbf{r})$. Ideally, approximations within DFT are made directly at the level of the free energy, which is a physically intuitive quantity. Correlation functions of all order can then be generated by successive functional differentiation. This is to be contrasted with standard integral equation approaches for which the closure is usually introduced at the level of the pair correlation functions, and which often make no direct reference to, or guarantee the existence of, an explicit generating functional. In practice, the distinction between integral equation and DFT approaches is frequently less clear-cut and many approximate DFTs rely on correlation functions obtained from integral equation theories as input.

The majority of modern density functional approaches are weighted density approximations in which the inhomogeneous density distributions are smoothed by some physically motivated set of weight functions [28]. Using cluster expansion methods [29] the exact excess Helmholtz free energy density can be expressed as a power series in the inhomogeneous density fields, $\rho_{i}(\mathbf{r})$. For the WR model, truncation of this series at $\mathcal{O}\left(\rho^{2}\right)$ recovers the original MF theory [1, 2]. The only diagram contributing to $\mathcal{O}\left(\rho^{3}\right)$ is the triangle diagram consisting of two root points and a single field point, all connected by Mayer bonds. As the root and field points cannot be labelled according to species without either an A-A or B-B Mayer bond occuring, the diagram is equal to zero. 
The first correction to the MF theory comes from the term $\mathcal{O}\left(\rho^{4}\right)$, which contains only one diagram. By neglecting terms $\mathcal{O}\left(\rho^{5}\right)$ and higher we obtain the following simple approximation

$$
\beta \mathcal{F}^{e x}\left[\rho_{A}, \rho_{B}\right]=-\bullet-\longrightarrow
$$

where black and gray field particles are associated with the density fields $\rho_{A}(\mathbf{r})$ and $\rho_{B}(\mathbf{r})$, respectively, and are connected by Mayer bonds. Note that the above diagrams are unlabeled. To convert to labeled diagrams requires multiplication by the appropriate prefactor (1 and $1 / 4)$.

At this point we draw on the experience of previous virial expansion studies of the WR model [10, 11, 12, 13] and take the truncated expansion (3) as the generating functional for our correlation functions, at least to a first level of approximation. We argue that the above two diagrams contain the dominant structural elements (basis functions) for an accurate description of the WR model at all densities. Our reasons for this assertion are the following: (i) investigations of the WR virial series suggest that inclusion of higher order diagrams worsens the description of the thermodynamic properties, (ii) the pair direct correlations and radial distribution functions generated from the $\mathrm{OZ}$ route give a reasonable account of existing simulation results at the simulated statepoints (see below), (iii) it can be proven that the MF theory becomes exact in the limit of infinite dimension [30]. The key part of the proof rests on identification of the four field particle diagram as the numerically dominant correction term to the MF theory.

It should be emphasized that although Eq. (3) provides a reasonable approximation for the thermodynamic functions over a portion of the phase diagram, the functional (Eq.(3)) is not a good theory for the phase boundary and is not intended as such. Construction of a functional which accurately predicts both the bulk binodal and inhomogeneous structure is a lofty goal which we do not pursue in the present work (see [16] for work in this direction). Here we present Eq.(3) as a means to obtain closures at the pair correlation level which may be subsequently modified and improved. The bulk free energy obtained from the uniform density limit of Eq.(3) is given by

$$
\frac{\beta F^{e x}}{V}=\frac{4}{3} \pi \rho_{A} \rho_{B}-\frac{34816}{181440} \pi^{3} \rho_{A}^{2} \rho_{B}^{2} .
$$

The total Helmholtz free energy, $\beta F / V=\rho \log (\rho)-\rho+$ $\rho x \log (x)+\rho(1-x) \log (1-x)+\beta F^{e x} / V$, displays two van der Waals loops as a function of $x$ for sufficiently large values of $\rho$ and is thus unable to account for the demixing transition.

Within DFT the bulk pair correlation functions may be obtained using either the test-particle route (minimizing the functional in the external field due to a particle fixed at the origin) or the Ornstein-Zernike (OZ) route. Following the $\mathrm{OZ}$ route the inhomogeneous pair direct
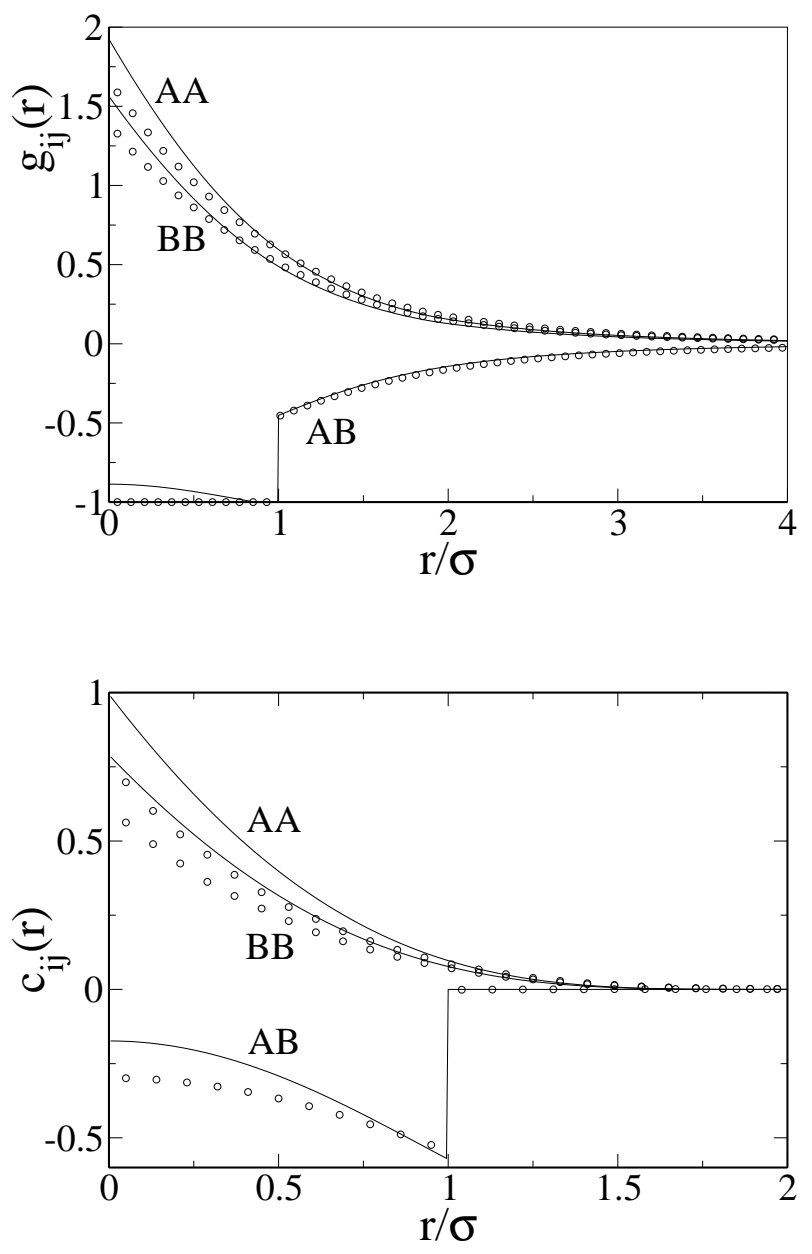

FIG. 7: The total and direct correlation functions for statepoint 2. Lines are the results of Eqs. (6)- (9). Circles are the simulation results.

correlation functions are obtained by taking two functional derivatives of the excess free energy functional

$$
c_{i j}\left(\mathbf{r}_{1}, \mathbf{r}_{2}\right)=-\beta \frac{\delta^{2} \mathcal{F}^{e x}\left[\left\{\rho_{i}\right\}\right]}{\delta \rho_{i}\left(\mathbf{r}_{1}\right) \delta \rho_{j}\left(\mathbf{r}_{2}\right)} .
$$

The homogeneous limit is then taken, $c_{i j}\left(\mathbf{r}_{1}, \mathbf{r}_{2}\right) \rightarrow$ $c_{i j}\left(r_{12}\right)$, and the bulk direct correlation functions are substituted into the OZ relations to yield the radial distribution functions. For a binary fluid the OZ relations for the homogeneous fluid are given by

$$
\tilde{h}_{i j}(k)=\tilde{c}_{i j}(k)+\sum_{l} \rho_{l} \tilde{c}_{i l}(k) \tilde{h}_{l j}(k),
$$

where the tilde denotes a Fourier transform. Application of this prescription to the functional (3) generates the following simple expressions for the bulk direct correlation functions

$$
\begin{aligned}
& c_{A B}(r)=f(r)+\rho_{A} \rho_{B} f(r) t_{2}(r) \\
& c_{A A}(r)=\rho_{B}^{2} t_{1}^{2}(r) / 2 \\
& c_{B B}(r)=\rho_{A}^{2} t_{1}^{2}(r) / 2,
\end{aligned}
$$



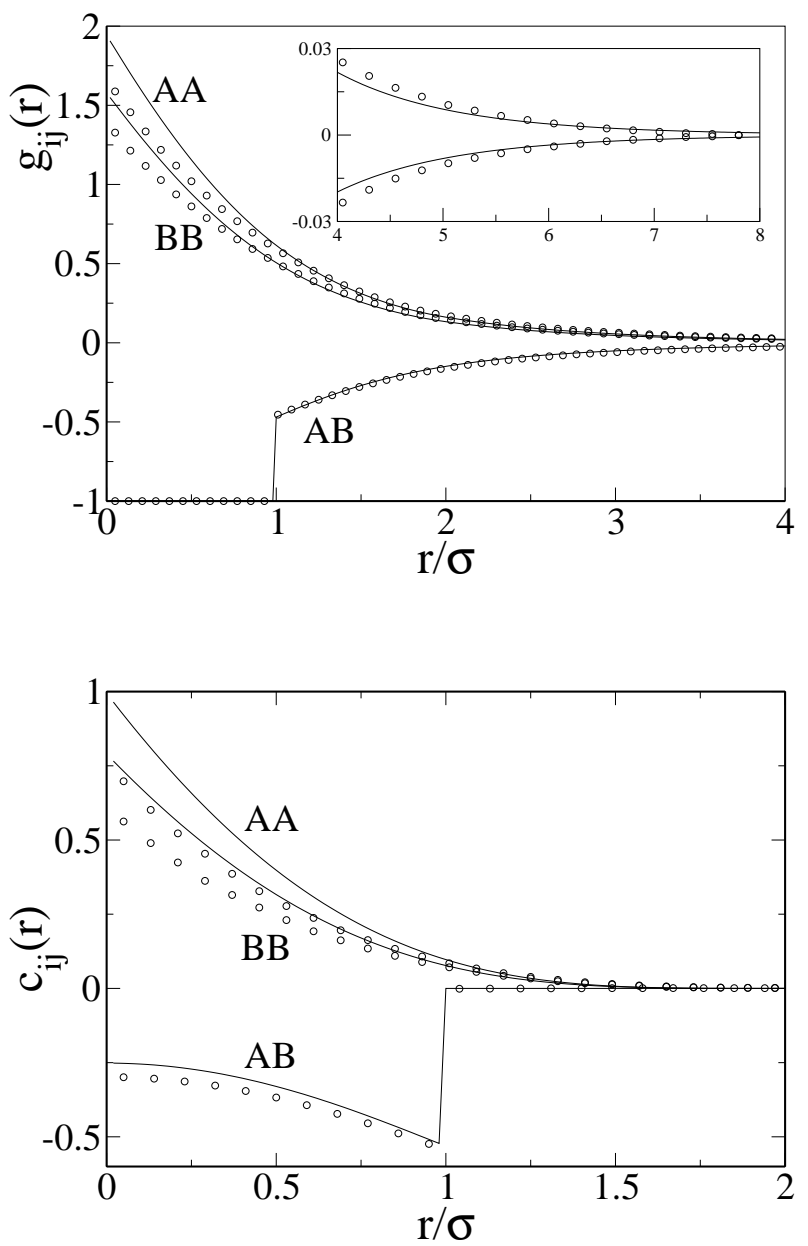

FIG. 8: The total and direct correlation functions for statepoint 2, close to the symmetry line. Lines are the results of closure Eq.(12). Circles are the simulation results.

where $f(r)$ is the Mayer function, $f(r)=-1$ for $r<1$, $f(r)=0$ for $r>1$, and $t_{1}(r)$ and $t_{2}(r)$ are the two lowest order chain diagrams given by

$$
\begin{aligned}
& t_{1}(r)=\frac{4}{3} \pi\left(1-\frac{3}{4} r+\frac{1}{16} r^{3}\right) r \\
& t_{2}(r)=-\left(\frac{\pi^{2}}{5040}\right)\left(r^{3}+12 r^{2}+27 r-6\right)(r-3)^{4} \\
& r \leq 3
\end{aligned}
$$

and zero otherwise. Fig. 7 compares the pair correlation functions obtained from Eqs.(6)-(9) with the simulation results for statepoint 2 . The level of agreement with the simulation $g_{i j}(r)$ at such a high density $\left(\rho \approx 0.85 \rho_{\text {crit }}\right)$ is surprising, given the fact that the correlation functions are generated from a truncated density expansion. In particular, the calculated $g_{A B}(r>1)$ lies very close to the simulation results. Although the general features of the $c_{i j}(r)$ are captured, the overall level of agreement is less satisfactory than for the $g_{i j}(r)$.

\section{B. Imposing the core condition}

The most obvious deficiency of the present approach is the violation of the exact core condition, $h_{A B}(r)=-1$ for $r<1$. Violation of this condition is a general drawback of the OZ route in DFT studies and only in very special cases, e.g. the Rosenfeld functional for additive hard sphere mixtures [31], is the core condition exactly satisfied. However, as we are primarily interested in the pair correlations this difficulty is easily resolved by replacing the closed form expression Eq.(7) for $c_{A B}(r)$ with a relation which enforces the core condition. Since our simulation results strongly suggest the approximation $c_{A B}(r>1)=0$, and given that this condition is already satisfied by Eq.(7), we are led to suggest the following relations

$$
\begin{aligned}
& h_{A B}(r)=-1 \quad r<1 \\
& c_{A B}(r)=0 \quad r \geq 1 \\
& c_{A A}(r)=\rho_{B}^{2} t_{1}^{2}(r) / 2 \\
& c_{B B}(r)=\rho_{A}^{2} t_{1}^{2}(r) / 2 .
\end{aligned}
$$

Combined with the OZ relation, Eq.(6), this leads to a closed theory for the pair correlation functions. These relations correspond to a linearization of the expressions for $c_{A A}(r)$ and $c_{B B}(r)$ in the YS integral equation [14].

In Fig. 8 we show some results obtained using closure (12) at the same statepoint shown in Fig. (7) Eq.(12) was solved using standard iterative numerical methods. Imposing the core condition leads to a distinct improvement upon the closed form expression Eq.(77). The functions $c_{A A}(r)$ and $c_{B B}(r)$ are identical to those shown in Fig. 7. but $c_{A B}(r)$ now lies considerably closer to the simulation result. The functions $g_{A A}(r)$ and $g_{B B}(r)$ remain in error for small separations, but the level of agreement for $r>1$ is improved. There are also small corrections to the function $g_{A B}(r)$ over the entire range. We note that by imposing the core condition we are effectively incorporating many more diagrams (in principle, an infinite number) into our description of the pair correlations. The price we pay for going beyond the simple virial approach of (31) is that we must resort to fully numerical solution.

\section{Spinodal line and critical point}

As $\rho$ is increased for fixed $x$, the partial structure factors $S_{i j}(k=0)$ diverge at a well defined point. The locus of these points defines the spinodal line which divides the phase diagram into regions of mechanical stability and instability. The minimum of this curve, located at $x=1 / 2$ for the present model, identifies the critical point. It is well known that approximate integral equations often fail to exhibit a true spinodal but yield instead a no-solutions region in the phase diagram within which the theory simply fails to converge (see Ref. 32 and references therein). Indeed, the study of Shrew and Yethiraj [4] performed 
on the symmetry line, and our own investigations for offsymmetry compositions, strongly suggest that all standard closures, with the exception of the mean-field and Percus-Yevick theories, fail to exhibit diverging structure factors prior to breakdown of the theory, and are therefore incapable of making any comment regarding the region in the vicinity of the critical point.

In Fig. 1, we show the spinodal resulting from the closure of Eq. (12). For comparison, we also show the critical point from the mean-field theory [1, 2] and that from the density functional theory of [16]. The critical point predicted by Eq.(12) lies remarkably close to the simulation result. We find $\rho_{\text {crit }}=0.762$ which compares very favourably with the best current simulation estimates $\rho_{\text {crit }}=0.7470(8)$ [7] and $\rho_{\text {crit }}=0.7486 \pm 0.0002$ [9]. This represents a substantial improvement upon previous theoretical treatments. The numerical solution of Eq.(12) for points of high compressibility (i.e. close to the spinodal) deserves some additional comment. It is a general difficulty of standard numerical methods based on Eq. (6) that, upon approaching the critical point, the diverging correlation length renders inadequate methods requiring truncation of $h_{i j}(r)$ at some finite range $R$. The finite size effects which result from such truncation give rise to considerable difficulties when attempting to numerically assess the critical behaviour of a given integral equation 32]. These difficulties have been overcome for one-component fluids and mixtures with additive interactions using specialized algorithms [32]. However, these methods do not generalize easily to non-additive mixtures, such as the WR model, and we have thus resorted to more traditional methods of iterative solution [33]. For this reason we make no definite claims regarding the critical exponents of the present theory; this would require a detailed study using specially tailored algorithms which goes beyond the scope of the present work. However, the numerical methods we have employed are certainly capable of unambiguous determination of the spinodal line. This enables us to confirm that the locus of points which we have identified is indeed a true spinodal and not simply a region of non-convergence. Although we refrain from making final claims regarding the nature of the criticality in our equations, we do make the observation that the spinodal is distinctly flatter in the vicinity of the critical point, compared to the mean-field approaches of $[1,2,16]$ or the PY theory. This may indicate interesting non-classical behaviour, and certainly warrants further investigation.

\section{CONCLUSIONS}

Using a combination of computer simulation and theoretical methods we have developed an integral equation for the WR model which yields good results for the pair structure and predicts the location of the critical point to an accuracy of approximately $2 \%$. This represents a considerable improvement upon previous theories which exhibit errors in the range $30-50 \%$. Our quasi-grand canonical computer simulations provide the first detailed information regarding the pair structure of the WR model for statepoints off the symmetry line $(x \neq 1 / 2)$ and provide confirmation that the condition $c_{i j}(r>1)=0$ is satisfied to a good level of approximation over the entire one-phase region. The integral equation here developed is very simple to use and requires no more numerical effort than solving standard integral equations such as PY or HNC. Our choice of basis functions for $c_{i i}(r)$ do leave some room for improvement, albeit at the cost of increased numerical effort. A more sophisticated scheme could involve basis functions with a free parameter, to be determined by enforcing thermodynamic consistency between virial and fluctuation equations of state. Considerable success was achieved in the case of additive hard sphere mixtures by constructing an approximation for $c_{i j}(r)$ using basis functions taken from the low order diagrams in the virial expansion [34]. The size of the field particle was treated as a parameter and scaled to interpolate between known low and high density limits. Whether a similar procedure is also feasible for the WR model remains an open question. Following completion of this work we were made aware of a very recent study in which a triplet level integral equation closure was applied to the WR model [35]. This approach is significantly more complicated than that followed in the present work but seems to yield very promising results worthy of further investigation.

\section{Acknowledgments}

This work was supported by the Deutsche Forschungsgemeinschaft under the SFB-TR6 (project sections A6 and D3).
[1] B. Widom and J.S. Rowlinson, J.Chem.Phys. 521670 (1969).

[2] J.S. Rowlinson and B. Widom, Molecular theory of capillarity, (Clarendon press, Oxford) (1982).

[3] S.N. Lai and M.E. Fisher, Mol.Phys. 881373 (1996); R. Dickman and G. Stell, J. Chem. Phys. 1028674 (1995).
[4] C.Y. Shew and A. Yethiraj, J.Chem.Phys. 1047665 (1996).

[5] G. Johnson, H. Gould, J. Machta and L.K. Chayes, Phys.Rev.Lett. 792612 (1997).

[6] W.T. Góźdź, J.Chem.Phys. 122074505 (2005).

[7] J. Buhot, J.Chem.Phys. 122024105 (2005).

[8] R. Fantoni and G. Pastore, Physica A 332349 (2004). 
[9] R.L.C. Vink, J. Chem. Phys. 124094502 (2006).

[10] T.W. Melnyk, J.S. Rowlinson and B.L. Sawford, Mol.Phys. 24809 (1972).

[11] J.P. Straley, M.A. Cotter, T.J. Lie and B. Widom, J.Chem.Phys. 574484 (1972).

[12] E. Helfand and F.H. Stillinger, J.Chem.Phys. 491232 (1968).

[13] M.I. Guerrero, J.S. Rowlinson and B.L. Sawford, Mol.Phys. 281603 (1974).

[14] A. Yethiraj and G. Stell, J.Stat.Phys. 10039 (2000).

[15] K. Jagannathen, G. Reddy and A. Yethiraj, 1096764 (2005).

[16] M. Schmidt, Phys.Rev.E. 63 010101(R) (2001).

[17] S. Ahn and J.L.Lebowitz, Phys.Lett. 44A 424 (1973); S. Ahn and J.L.Lebowitz, J.Chem.Phys. 60523 (1974).

[18] Our numerical solutions of the PY equation confirm the classical character of the PY critical point for the WR model, in accord with the findings of [17]. We note that the PY condition $c_{i i}(r)=0$ allows the binary OZ equations to be reduced to a single $\mathrm{OZ}$ equation of standard form. An adaptation of the numerical method presented in 32] can then be applied to study the critical region.

[19] M. E. Fisher and S.-Y. Zinn, J. Phys. A: Math. Gen. 31 L629 (1998).

[20] M. E. Fisher, Phys. Rev. 176257 (1968).

[21] Ruichao Ren, C. J. O'Keeffe, and G. Orkoulas, Scaling Fields and Pressure Mixing in the Widom-Rowlinson Model, submitted (2006).
[22] R.L.C. Vink and J. Horbach, J. Chem. Phys. 1213253 (2004).

[23] R.L.C. Vink, Entropy Driven Phase Separation in a Model Colloid-Polymer Mixture, cond-mat/0402585 (2004).

[24] M. P. Allen and D. J. Tildesley, Computer Simulation of Liquids, Oxford University Press (Oxford, England, 1989).

[25] M.E. Fisher, J. Math. Phys. 5, 944 (1964).

[26] J. Kolafa, S. Labik and A. Malijevsky, Mol. Phys. 100, 2629 (2002).

[27] Y. Rosenfeld and N.W. Ashcroft, Phys. Rev. A 20, 1208 (1979).

[28] R. Evans, in Fundamentals of inhomogeneous fluids, ed. D. Henderson, Ch.2 (Dekker, New York, 1992).

[29] G. Stell, in The equilibrium theory of classical fluids, eds. H.L. Frisch and J.L. Lebowitz, p.171 (Benjamin, New York, 1969).

[30] T.J. Lie, J.Chem.Phys. 56332 (1972).

[31] Y. Rosenfeld, Phys.Rev.Lett. 63980 (1989).

[32] J.M. Brader, Int.J.Thermophys. 27394 (2006).

[33] J.P. Hansen and I.R. McDonald, Theory of simple liquids, (Academic press, London, 1986).

[34] Y. Rosenfeld, J. Chem. Phys. 894272 (1988).

[35] A. Malijevsky, S. Sokolowsky and T. Zientarski, J. Chem. Phys. 125114505 (2006). 